\title{
COMPLETENESS IN FINITE ALGEBRAS WITH A SINGLE OPERATION
}

\section{G. ROUSSEAU}

An algebra $\left\langle A,\left(\omega_{i}\right)_{i \in I}\right\rangle$ is said to be complete if for each $n$ all functions $f: A^{n} \rightarrow A$ are definable in terms of the operations $\omega_{i}(i \in I)$. The purpose of this note is to show that a finite algebra $\langle A, \omega\rangle$ with a single operation is complete if and only if it has no subalgebras, automorphisms or homomorphic images apart from the obvious trivial ones. This generalizes results of Post [1] $(|A|=2)$ and Wheeler [2] $(|A|=3)$. The proof is based on a theorem of Rosenberg [3] which characterizes complete finite algebras with any number of operations; the latter theorem in turn generalizes results of Post [1] $(|A|=2)$ and Jablonskiǐ $[4](|A|=3){ }^{1}$

As a corollary of the main result we show that a finite algebra $\langle A, \omega\rangle$ with more than two elements is complete iff $\omega$ generates a doubly transitive subgroup of the symmetric group on $A$. This improves results of Salomaa [5] and Schofield [7].

In order to state Rosenberg's theorem we need to introduce certain definitions. For $k>0$ we denote by $E_{k}$ the set $\{0,1, \cdots, k-1\}$. An $h$-ary relation is a subset $R$ of $E_{k}^{h}$, and is called universal if $R=E_{k}^{h}$; we write $R\left(x_{1}, \cdots, x_{h}\right)$ when $\left(x_{1}, \cdots, x_{h}\right) \in R$. If $f$ is a function of $n$ arguments and $R$ is an $h$-ary relation we say that $f$ preserves $R$ if $R\left(f\left(x_{1}^{1}, \cdots, x_{1}^{n}\right), \cdots, f\left(x_{h}^{1}, \cdots, x_{h}^{n}\right)\right)$ whenever $R\left(x_{1}^{1}, \cdots, x_{h}^{1}\right)$, $\cdots, R\left(x_{1}^{n}, \cdots, x_{h}^{n}\right)$.

An $h$-ary relation is totally reflexive if $R\left(x_{1}, \cdots, x_{h}\right)$ whenever $x_{1}, \cdots, x_{h}$ are not all distinct, and totally symmetric if for each permutation $\sigma$ of the integers $1, \cdots, h, R\left(x_{1}, \cdots, x_{h}\right) \Leftrightarrow R\left(x_{\sigma(1)}, \cdots, x_{\sigma(h)}\right)$. The centre of a totally symmetric $h$-ary relation is the set of elements $c$ such that $R\left(x_{1}, \cdots, x_{h-1}, c\right)$ holds for all $x_{1}, \cdots, x_{h-1} \in E_{k}$. A relation is said to be central if it is totally reflexive and totally symmetric and has centre $C, \varnothing \subset C \subset E_{k}$. Thus the singularly central relations are just the proper nonnull subsets of $E_{k}$. For $a \in E_{h}{ }^{m}$ we denote by $[a]_{l}$ the $l$ th digit $(l=0, \cdots, m-1)$ in the expansion $a=\sum_{l=0}^{m-1}[a]_{l} h^{l}$ of $a$ in the scale of $h$.

We may now state the theorem of Rosenberg as follows:

Received by the editors September 5, 1966.

${ }^{1}$ Dr. Roy O. Davies kindly communicated to me a derivation of Wheeler's result from the theorem of Jablonskil and remarked that he had established Theorem 1 for $|A| \leqq 8$ using Rosenberg's theorem (the proof of which has not yet been published). 
For a finite algebra $\left\langle E_{k},\left(\omega_{i}\right)_{i \in I}\right\rangle(k>2)$ to be complete it is necessary and sufficient that in each of the following cases there exists at least one $\omega_{i}(i \in I)$ that does not preserve the relation $R$ :

$1^{\circ}$. $R$ is a partial order on $E_{k}$ with greatest and least element.

$2^{\circ}$. $R$ is (the graph of) a nonidentical permutation of $E_{k}$.

$3^{\circ} . R$ is the relation $\left\langle\left\langle x_{1}+x_{2}=x_{3}+x_{4}\right\rangle\right\rangle$ where $k=p^{m}$ and $G=\left\langle E_{k},+\right\rangle$ is an abelian group in which each nonzero element has order $p$.

$4^{\circ} . R$ is a nonidentical, nonuniversal equivalence relation on $E_{k}$.

$5^{\circ} . R$ is an h-ary central relation on $E_{k}(1 \leqq h \leqq k)$.

$6^{\circ} . R$ is for some $h(3 \leqq h \leqq k)$ and some surjection $\phi: E_{k} \rightarrow E_{h^{m}}$ $(m>0)$ the h-ary relation

$$
\left|\left\{\left[\phi\left(x_{1}\right)\right]_{l}, \cdots,\left[\phi\left(x_{h}\right)\right]_{l}\right\}\right|<h \quad \text { for } l=0, \cdots, m-1
$$

(i.e., for each $l(l=0, \cdots, m-1)$ at least two of the numbers $\phi\left(x_{1}\right)$, $\cdots, \phi\left(x_{h}\right)$ have the same lth digit when expressed in the scale of $\left.h\right)$.

If $\left\langle A,\left(\omega_{i}\right)_{i \in I}\right\rangle$ is an arbitrary algebra, then a nonempty subset of $A$, a permutation of $A$, or an equivalence on $A$ is called a subalgebra, automorphism or congruence respectively iff it is preserved by each $\omega_{i}(i \in I)$. A subalgebra distinct from $A$ is said to be proper, as is an automorphism other than the identity, or a nonuniversal, nonidentical congruence. The existence of proper congruences on $A$ is equivalent to the existence of homomorphic images of $A$ not isomorphic to $A$ or to the one-element algebra.

Theorem 1. A finite algebra $\langle A, \omega\rangle$ with a single operation is complete if and only if it has no proper subalgebras, automorphisms or congruences.

Proof. In view of the above-mentioned result of Post we may restrict attention to the case $A=E_{k}, k>2$. The necessity of the condition is clear from $2^{\circ}, 4^{\circ}, 5^{\circ}$ of Rosenberg's theorem. To prove sufficiency we have to show in cases $1^{\circ}-6^{\circ}$ that if $\omega$ preserves $R$ then $A$ has a proper subalgebra, automorphism or congruence.

$1^{\circ}$. If $\omega$ preserves a partial order $\leqq$ with greatest element $u$, then either $\{u\}$ or $A \backslash\{u\}$ is a proper subalgebra. For, if $\omega\left(x_{1}, \cdots, x_{n}\right)=u$ for some $x_{1}, \cdots, x_{n} \in A \backslash\{u\}$ then, because $x_{1} \leqq u, \cdots, x_{n} \leqq u$, we have $u=\omega\left(x_{1}, \cdots, x_{n}\right) \leqq \omega(u, \cdots, u)$ so that $\omega(u, \cdots, u)=u$.

$3^{\circ}$. Suppose $k=p^{m}$ and $G=\left\langle E_{k},+\right\rangle$ is an abelian group in which each nonzero element has order $p$. Then $G$, being the direct sum of $m$ cyclic groups of order $p$, may be identified with the space $\left(Z_{p}\right)^{m}$ where $Z_{p}$ is the residue class field of the integers modulo $p$. If $\omega$ preserves the relation $\left\langle\left\langle x_{1}+x_{2}=x_{3}+x_{4}\right\rangle\right\rangle$ then it satisfies the functional equation 
$\omega\left(x_{1}, \cdots, x_{n}\right)+\omega\left(y_{1}, \cdots, y_{n}\right)=\omega\left(x_{1}+y_{1}, \cdots, x_{n}+y_{n}\right)+\omega(0, \cdots, 0)$

and it is not difficult to show that the solutions are of the form

$$
\omega\left(x_{1}, \cdots, x_{n}\right)=a+x_{1} A_{1}+\cdots+x_{n} A_{n}
$$

where $a \in\left(Z_{p}\right)^{m}$ and $A_{1}, \cdots, A_{n}$ are $m \times m$ matrices over $Z_{p}$. If $\operatorname{det}\left(I-\sum A_{i}\right)=0$ then the equation $0=x\left(I-\sum A_{i}\right)$ has a nonzero solution $x=x_{0}$, so that

$$
\omega\left(x_{0}+x_{1}, \cdots, x_{0}+x_{n}\right)=x_{0}+\omega\left(x_{1}, \cdots, x_{n}\right) ;
$$

i.e. $x \rightarrow x_{0}+x$ is a proper automorphism. If $\operatorname{det}\left(I-\sum A_{i}\right) \neq 0$ then the equation $a=x\left(I-\sum A_{i}\right)$ has a solution $x=x_{0}$, so that $\omega\left(x_{0}, \cdots, x_{0}\right)$ $=x_{0}$; i.e. $\left\{x_{0}\right\}$ is a proper subalgebra.

$5^{\circ}$. Suppose $\omega$ preserves the $h$-ary central relation $R$. Let $C_{0}$ be the centre of $R$ and for each $\nu$ let $C_{\nu+1}=C_{\nu} \cup \omega\left(C_{\nu}^{n}\right)$; we show that $C_{\nu}^{h} \subseteq R$ for each $\nu$. This is clear for $\nu=0$; suppose $C_{\nu}^{h} \subseteq R,\left(x_{1}, \cdots, x_{h}\right)$ $\in C_{\nu+1}^{h}$. Then $\left(x_{1}, \cdots, x_{h}\right) \in R$ if some $x_{i} \in C_{0}$. If no $x_{i} \in C_{0}$ we may write $x_{i}=\omega\left(x_{i}^{1}, \cdots, x_{i}^{n}\right)$ with $\left(x_{i}^{1}, \cdots, x_{i}^{n}\right) \in C_{\nu}^{n}(i=1, \cdots, h)$, so again $\left(x_{1}, \cdots, x_{h}\right) \in R$ since by induction hypothesis $R\left(x_{1}^{1}, \cdots, x_{h}^{1}\right)$, $\cdots, R\left(x_{1}^{n}, \cdots, x_{h}^{n}\right)$. Since $R$ is nonuniversal it follows from what has been proved that $\mathrm{U}_{\nu \geqq 0} C_{\nu} \neq E_{k}$; thus $\mathrm{U}_{\nu \geqq 0} C_{\nu}$ is a proper subalgebra.

$6^{\circ}$. Suppose $\omega$ preserves the $h$-ary relation $R$, where $3 \leqq h \leqq k$ and for some surjection $\phi: E_{k} \rightarrow E_{h}{ }^{m}(m>0), R\left(x_{1}, \cdots, x_{h}\right)$ is equivalent to

$$
\left|\left\{\left[\phi\left(x_{1}\right)\right]_{l}, \cdots,\left[\phi\left(x_{h}\right)\right]_{l}\right\}\right|<h \quad \text { for } l=0, \cdots, m-1 .
$$

(a) If $h^{m}<k$ then $\left\langle\left\langle\phi\left(x_{1}\right)=\phi\left(x_{2}\right)\right\rangle\right\rangle$ is a proper congruence unless $E_{k}$ has a proper subalgebra. For, $\left\langle\left\langle\phi\left(x_{1}\right)=\phi\left(x_{2}\right)\right\rangle\right\rangle$ is a nonidentical, nonuniversal equivalence relation; we show that $\phi\left(x_{1}^{1}\right)=\phi\left(x_{2}^{1}\right), \cdots$, $\phi\left(x_{1}^{n}\right)=\phi\left(x_{2}^{n}\right)$ imply $\phi\left(\omega\left(x_{1}^{1}, \cdots, x_{1}^{n}\right)\right)=\phi\left(\omega\left(x_{2}^{1}, \cdots, x_{2}^{n}\right)\right)$. Suppose the contrary. For some $l(l=0, \cdots, m-1),\left[\phi\left(\omega\left(x^{1}, \cdots, x^{n}\right)\right)\right]_{l}$ $\neq\left[\phi\left(\omega\left(x_{2}^{1}, \cdots, x_{2}^{n}\right)\right)\right]_{l}$. If $E_{k}$ has no proper subalgebra the mapping $\omega: E_{k}^{n} \rightarrow E_{k}$ is surjective, as are $\phi: E_{k} \rightarrow E_{h^{m}}$ and $[\cdots]_{l}: E_{h^{m} \rightarrow E_{h}}$. Thus elements $x_{i}^{1}, \cdots, x_{i}^{n}(i=3, \cdots, h)$ may be chosen so that $\left[\phi\left(\omega\left(x_{i}^{1}, \cdots, x_{i}^{n}\right)\right)\right]_{l}(i=1, \cdots, h)$ are all distinct, i.e. $R\left(\omega\left(x_{1}^{1}, \cdots, x_{1}^{n}\right), \cdots, \omega\left(x_{h}^{1}, \cdots, x_{h}^{n}\right)\right)$ does not hold. Since $R\left(x_{1}^{1}, \cdots, x_{h}^{1}\right), \cdots, R\left(x_{1}^{n}, \cdots, x_{h}^{n}\right)$ hold, this contradicts the supposition that $\omega$ preserves $R$.

(b) Suppose $h^{m}=k$ so that $\phi$ is bijective. Since a function $f\left(x^{1}, \cdots, x^{n}\right)$ preserves a relation $S\left(y_{1}, \cdots, y_{h}\right)$ iff $\phi\left(f\left(\phi^{-1}\left(x^{1}\right), \cdots\right.\right.$, $\left.\left.\phi^{-1}\left(x^{n}\right)\right)\right)$ preserves $S\left(\phi^{-1}\left(y_{1}\right), \cdots, \phi^{-1}\left(y_{h}\right)\right)$ it is easy to see that it suffices to consider the case where $\phi$ is the identity: i.e. $R$ is the relation 


$$
\left|\left\{\left[x_{1}\right]_{l}, \cdots,\left[x_{h}\right]_{l}\right\}\right|<h \quad \text { for } l=0, \cdots, m-1 .
$$

Consider the mappings $g_{l}: E_{h}^{m n} \rightarrow E_{h}(l=0, \cdots, m-1)$ defined by

$$
\begin{aligned}
g_{l}\left(\xi_{0}^{1}, \cdots, \xi_{m-1}^{1} ; \cdots ; \xi_{0}^{n}, \cdots, \xi_{m-1}^{n}\right) \\
=\left[\omega\left(\sum_{j=0}^{m-1} \xi_{j}^{1} h^{j}, \cdots, \sum_{j=0}^{m-1} \xi_{j}^{n} h^{j}\right)\right]_{l .}
\end{aligned}
$$

We may suppose each $g_{l}$ surjective for otherwise $E_{k}$ has a proper subalgebra. If some $g_{l}$ depends essentially on more than one of its arguments then (cf. [4], [6], [8]) we can find $(h-1)$-element subsets $H_{1}, \cdots, H_{m n}$ of $E_{h}$ such that $g_{l}$ assumes $h$ values on $H_{1} \times \cdots \times H_{m n}$; but this would imply that $\omega$ does not preserve $R$. Thus each $g_{l}$ $(l=0, \cdots, m-1)$ depends essentially on only one argument, so we may write

$g_{l}\left(\xi_{0}^{1}, \cdots, \xi_{m-1}^{1} ; \cdots ; \xi_{0}^{n}, \cdots, \xi_{m-1}^{n}\right)=\gamma_{l}\left(\xi_{\tau(l)}^{\sigma(l)}\right) \quad(l=0, \cdots, m-1)$

and

$$
\omega\left(x^{1}, \cdots, x^{n}\right)=\sum_{l=0}^{m-1} \gamma_{l}\left(\left[x^{\sigma(l)}\right]_{\tau(l)}\right) h^{l} .
$$

If some proper nonnull subset $I$ of the indices $0, \cdots, m-1$ is invariant under the mapping $\tau(\tau(I) \subseteq I)$, then the relation $\left\langle\left\langle[x]_{i}=[y]_{i}\right.\right.$ for each $\left.\left.i \in I\right\rangle\right\rangle$ is a proper congruence. In the contrary case $\tau$ is a permutation of $0, \cdots, m-1$ consisting of a single cycle of length $m$. Set

$$
\alpha(x)=\omega(x, \cdots, x)=\sum_{l=0}^{m-1} \gamma_{l}\left([x]_{\tau(l)}\right) h^{l} .
$$

By induction we have

$$
\alpha^{r}(x)=\sum_{l=0}^{m-1} \gamma_{l} \gamma_{\tau^{(l)}} \cdots \gamma_{\tau^{r-1}(l)}\left([x]_{\tau^{r}(l)}\right) h^{l},
$$

so, in particular,

$$
\alpha^{m}(x)=\sum_{l=0}^{m-1} \gamma_{l} \gamma_{\tau(l)} \cdots \gamma_{\tau^{m-1}(l)}\left([x]_{l}\right) h^{l} .
$$

From this we verify that

$$
\alpha^{m}\left(\omega\left(x^{1}, \cdots, x^{n}\right)\right)=\omega\left(\alpha^{m}\left(x^{1}\right), \cdots, \alpha^{m}\left(x^{n}\right)\right) .
$$

If $\alpha^{m}$ is not the identity we consider three cases: (i) if $\alpha^{m}$ is constant $=x_{0}$, then 


$$
\omega\left(x_{0}, \cdots, x_{0}\right)=\omega\left(\alpha^{m}\left(x_{0}\right), \cdots, \alpha^{m}\left(x_{0}\right)\right)=\alpha^{m}\left(\omega\left(x_{0}, \cdots, x_{0}\right)\right)=x_{0}
$$

so that $\left\{x_{0}\right\}$ is a proper subalgebra; (ii) if $\alpha^{m}$ is one-one then $\alpha^{m}$ is a proper automorphism; (iii) if $\alpha^{m}$ is not constant and not one-one, then $\left\langle\left\langle\alpha^{m}\left(x_{1}\right)=\alpha^{m}\left(x_{2}\right)\right\rangle\right\rangle$ is a proper congruence. If $\alpha^{m}$ is the identity then $\gamma_{l} \gamma_{\tau(l)} \cdots \gamma_{\tau^{m-1}(l)}$ is the identity on $E_{h}(l=0, \cdots, m-1)$. Choosing $\xi_{0} \in E_{h}$ arbitrarily we may set

$$
\xi_{r^{r}(0)}=\gamma_{r^{r}(0)} \cdots \gamma_{r^{m-1}(0)}\left(\xi_{0}\right) \quad(r=0, \cdots, m-1),
$$

so that $\gamma_{l}\left(\xi_{\tau(l)}\right)=\xi_{l}(l=0, \cdots, m-1)$; then if $x=\sum_{l=0}^{m-1} \xi_{l} h^{l}$ we have $\omega(x, \cdots, x)=x$, i.e. $\{x\}$ is a proper subalgebra.

The proof of Theorem 1 is now complete.

THEOREM 2. A finite algebra $\langle A, \omega\rangle$ with more than two elements is complete if and only if the set of functions definable from $\omega$ includes a doubly transitive subgroup of the symmetric group on $A$.

Proof. If $A$ is not complete then by Theorem 1 it has a proper subalgebra, automorphism or congruence, which must be preserved by all functions definable in terms of $\omega$. But in general if $R\left(a_{1}, \cdots, a_{h}\right)$ holds while $R\left(a_{1}^{\prime}, \cdots, a_{h}^{\prime}\right)$ does not, where $\left\{a_{1}, \cdots, a_{h}\right\}$ and $\left\{a_{1}^{\prime}, \cdots, a_{h}^{\prime}\right\}$ each consist of $h$ elements, then $R$ is not preserved by any permutation which carries $a_{1}$ to $a_{1}^{\prime}, \cdots, a_{h}$ to $a_{h}{ }^{\prime}$. Applying this in the case of a proper subalgebra, automorphism or congruence, where $h$ does not exceed 2 , it follows that $\omega$ cannot generate a doubly transitive group of permutations.

\section{REFERENCES}

1. E. L. Post, The two-valued iterative systems of mathematical logic, Princeton Univ. Press., Princeton, N. J., 1941.

2. R. F. Wheeler, Complete connectives for the 3-valued propositional calculus, Proc. London Math. Soc. (3) 16 (1966), 167-191.

3. I. Rosenberg, La structure des fonctions de plusieurs variables sur un ensemble fini, C. R. Acad. Sci. Paris 260 (1965), 3817-3819.

4. S. V. Jablonskir, Functional constructions in k-valued logic, Trudy Mat. Inst. Steklov 51 (1958), 5-142. (Russian)

5. A. Salomaa, On basic groups for the set of functions over a finite domain, Ann. Acad. Sci. Fenn. Ser. A I Math. 338 (1963), 1-15.

6. - On essential variables of functions, especially in the algebra of logic, ibid. 339 (1963), 1-11.

7. P. Schofield, Complete subsets of mappings over a finite domain, Proc. Cambridge Philos. Soc. 62 (1966), 597-611.

8. Roy O. Davies, Two theorems on essential variables, J. London Math. Soc. 41 (1966), 333-335.

UNiversity of Leicester, ENGLAND 